\title{
Quantum oscillations of self-dual Abrikosov-Nielsen-Olesen vortices
}

\author{
A. Alonso Izquierdo ${ }^{(1)}$, W. Garcia Fuertes ${ }^{(2)}$, M. de la Torre Mayado ${ }^{(3)}$ and J. Mateos Guilarte ${ }^{(4)}$ \\ (1) Departamento de Matematica Aplicada, Universidad de Salamanca, SPAIN \\ (3) Departamento de Fisica, Universidad de Salamanca, SPAIN \\ (4) Departamento de Fisica and IUFFYM, Universidad de Salamanca, SPAIN \\ (2) Departamento de Fisica, Universidad de Oviedo, SPAIN
}

\begin{abstract}
The mass shift induced by one-loop quantum fluctuations on self-dual ANO vortices is computed using heat kernel/generalized zeta function regularization methods. The quantum masses of superimposed multi-vortices with vorticity lower than five are given. The case of two separate vortices with a quantum of magnetic flux is also discussed.
\end{abstract}

PACS numbers: 03.70.+k,11.15.Kc,11.15.Ex

\section{INTRODUCTION}

In this paper we present some new results on the quantization of the self dual multi-vortex solutions of the Abelian Higgs model. We also take the opportunity to offer a detailed description of the concepts and techniques that allowed us to compute the one-loop quantum correction to the mass of self-dual Abrikosov-Nielsen-Olesen vortices with one quantum of magnetic flux in the Rapid Communication [1]. The AHM provides a theoretical ground in several fields of physics: it provides shape to interesting truncations of the electroweak or grand unified theories, it also provides the basis for the various phenomenological models for cosmic strings, or it can be used as a Ginzburg-Landau theory for superconductivity.

Interest in this research, developed in the supersymmetric framework in [2], 3], was rekindled two years ago. Non-vanishing quantum corrections to the mass of $N=2$ supersymmetric vortices were recently reported in papers [4] and [5], see also 27]. In the second paper, it was found that the central charge of the $N=2$ SUSY algebra receives a non-vanishing one-loop correction that is exactly equal to the one-loop mass shift; thus, one could talk in terms of one-loop BPS saturation. This result fits in a pattern first conjectured in [6] and then proved in [7] for supersymmetric kinks. Another work by the authors of the Stony Brook/Viena group, 8 8 unveils a similar kind of behavior of supersymmetric BPS monopoles in $N=2$ SUSY Yang-Mills theory. In this reference, however, it is pointed out that $(2+1)$-dimensional SUSY vortices do not behave exactly in the same way as their $(1+1)$ - and $(3+1)$-dimensional cousins. One-loop corrections in the vortex case are in no way related to an anomaly in the conformal central charge, contrarily to the quantum corrections for SUSY kinks and monopoles.

We shall focus, however, on the purely bosonic Abelian Higgs model and rely on the heat kernel/generalized zeta function regularization method that we developed in reference [9]. Our approach profits from the hightemperature expansion of the heat function, which is compatible with Dirichlet boundary conditions in purely bosonic theories. In contrast, the application of a similar regularization method to the supersymmetric kink requires SUSY-friendly boundary conditions, see [10]. In 9] the kink quantum correction in the $\phi^{4}$ model is estimated by this method and compared with the correct answer obtained from the Dashen-Hasslacher-Neveu formula, 11 in order to check the reliability of our approach. The relative error found is approximately $0.07 \%$. In 12 and 13 ] we also calculated the quantum mass corrections for kinks arising in two-component scalar models, where second-order small fluctuations are ruled by matrix differential operators. Therefore, we were led to generalize the zeta function method to the matrix case, because the DHN approach, based on a direct computation of the spectral density, is not efficient for matrix differential Schrodinger operators. This step has proved to be crucial, opening the possibility of applying our method to two-dimensional topological defects in the Abelian Higgs model.

In order to accomplish this task we shall encounter more difficulties than for one-dimensional multicomponent kinks. As noticed by Vassilevich, the lack of analytical expressions for vortex solutions forces us to perform a numerical analysis already at the classical level to solve the field equations. Also, the high-temperature expansion of the heat trace becomes more involved due to the jump from one to two spatial dimensions; the recurrence relations hold between partial -rather than ordinary- derivatives of the high-T expansion coefficients. We stress that the evaluation of the Seeley coefficients is a very laborious task: fluctuations of the vector, Higgs and Goldstone fields are governed by one $4 \times 4$-matrix differential operator, whereas fluctuations of the ghosts are determined by one scalar differential operator acting on $L^{2}\left(\mathbb{R}^{2}\right)$. There is, however, one point where the situation is more favorable as compared to the kink case: the generalized zeta function regularization method provides us directly with a finite quantity, without the need of infinite renormalizations. This fact is peculiar to even spatial dimensions and is probably related to the lack of anomalies when fermions are added. As for kinks, we shall obtain a simple formula for the one-loop quantum 
mass correction depending on the Seeley coefficients and the number of zero modes.

One remarkable aspect of our results is that the correction found by this method in the bosonic system is essentially twice the correction arising in the supersymmetric case in 44] and 5]. This seems to be in agreement with the relationship between the supersymmetric and non-supersymmetric one-loop corrections to the masses of the sine-Gordon and $\phi^{4}$ kinks, see 14] and 15].

The organization of the paper is as follows: In Section $\S .2$ we revise the perturbative sector of the Abelian Higgs model in the Feynman-'t Hooft renormalizable gauge and set the one-loop mass renormalization conventions. Section $\S .3$ is devoted to studying ANO vortex solutions and their fluctuations in a partially analytical, partially numerical manner. The high-temperature expansion of the pertinent heat traces is developed in Section $\S .4$. Section $\S .5$ explains how quantum oscillations of vortices are accounted for in the framework of generalized zeta function regularization. In Section $\S .6$ the one-loop vortex mass shift formula is applied to cylindrically symmetric self-dual vortices. We also briefly discuss how the shift depends on the distance between centers of a two-vortex solution. Finally, we offer a Summary and Outlook.

\section{THE PLANAR ABELIAN HIGGS MODEL}

\section{The model}

The AHM describes the minimal coupling between an $U(1)$-gauge field and a scalar field in a phase where the gauge symmetry is spontaneously broken. Defining nondimensional space-time variables, $x^{\mu} \rightarrow \frac{1}{e v} x^{\mu}$, and fields, $\phi \rightarrow v \phi=v\left(\phi_{1}+i \phi_{2}\right), A_{\mu} \rightarrow v A_{\mu}$, from the vacuum expectation value of the Higgs field $v$ and the $U(1)$-gauge coupling constant $e$, the action for the Abelian Higgs model in $(2+1)$-dimensions reads:

$$
S=\frac{v}{e} \int d^{3} x\left[-\frac{1}{4} F_{\mu \nu} F^{\mu \nu}+\frac{1}{2}\left(D_{\mu} \phi\right)^{*} D^{\mu} \phi-U\left(\phi, \phi^{*}\right)\right]
$$

with

$$
U\left(\phi, \phi^{*}\right)=\frac{\kappa^{2}}{8}\left(\phi^{*} \phi-1\right)^{2}
$$

$\kappa^{2}=\frac{\lambda}{e^{2}}$ is the only classically relevant parameter and measures the ratio between the square of the masses of the Higgs, $M^{2}=\lambda v^{2}$, and vector particles, $m^{2}=e^{2} v^{2}$; $\lambda$ is the Higgs field self-coupling. We choose a system of units where $c=1$, but $\hbar$ has dimensions of length $\times$ mass. Also, we define the metric tensor as: $g_{\mu \nu}=$ $\operatorname{diag}(1,-1,-1), \mu, \nu=0,1,2$.

\section{Feynman rules in the $R$-gauge}

The choice of $\phi^{V}=1$ as the ground state causes spontaneous symmetry breaking of the Abelian gauge invariance. In the Feynman-'t Hooft renormalizable gauge,

$$
R\left(A_{\mu}, G\right)=\partial_{\mu} A^{\mu}-G
$$

the particle spectrum involves a vector particle $A_{\mu}$, Higgs and Goldstone scalar particles $\phi=1+H+i G$, and a complex ghost $\chi$. The Feynman rules are read from the action, see Reference [16]:

$$
\begin{aligned}
S & =\frac{v}{e} \int d^{3} x\left[-\frac{1}{2} A_{\mu}\left[-g^{\mu \nu}\left(\partial_{\alpha} \partial^{\alpha}+1\right)\right] A_{\nu}\right. \\
& +\frac{1}{2} \partial_{\mu} G \partial^{\mu} G-\frac{1}{2} G^{2}+\frac{1}{2} \partial_{\mu} H \partial^{\mu} H-\frac{\kappa^{2}}{2} H^{2} \\
& +\partial_{\mu} \chi^{*} \partial^{\mu} \chi-\chi^{*} \chi-\frac{\kappa^{2}}{2} H\left(H^{2}+G^{2}\right) \\
& +A_{\mu}\left(\partial^{\mu} H G-\partial^{\mu} G H\right)+H\left(A_{\mu} A^{\mu}-\chi^{*} \chi\right) \\
& \left.-\frac{\kappa^{2}}{8}\left(H^{2}+G^{2}\right)^{2}+\frac{1}{2}\left(G^{2}+H^{2}\right) A_{\mu} A^{\mu}\right]
\end{aligned}
$$

\begin{tabular}{|c|c|c|c|}
\hline Particle & Field & Propagator & Diagram \\
\hline Higgs & $H(x)$ & $\frac{i e \hbar}{v\left(k^{2}-\kappa^{2}+i \varepsilon\right)}$ & . $\quad k$ \\
\hline Goldstone & $G(x)$ & $\frac{i e \hbar}{v\left(k^{2}-1+i \varepsilon\right)}$ & • \\
\hline Ghost & $\chi$ & $\frac{i e \hbar}{v\left(k^{2}-1+i \varepsilon\right)}$ & $\bullet-\stackrel{k}{\rightarrow}-\rightarrow$ \\
\hline Vector Boson & $A_{\mu}(x)$ & $\frac{-i e \hbar g^{\mu \nu}}{v\left(k^{2}-1+i \varepsilon\right)}$ & $\sim_{e_{\mu}}^{\sim^{k}} \sim_{e_{\nu}}$ \\
\hline
\end{tabular}

There are four propagators, plus five third-order and five fourth-order vertices shown in the next two Tables:

TABLE I: Propagators

\section{One-loop renormalization}

Defining

$$
I\left(c^{2}\right)=\int \frac{d^{3} k}{(2 \pi)^{3}} \cdot \frac{i}{k^{2}-c^{2}+i \varepsilon}
$$

and bearing in mind that $I\left(\kappa^{2}\right)=I(1)+$ finite part, the one-loop divergences in the planar Abelian Higgs model can be organized as follows: 
TABLE II: Third- and fourth-order vertices

Vertex Weight $-3 i \kappa^{2} \frac{v}{\hbar e}$

- Higgs tadpole

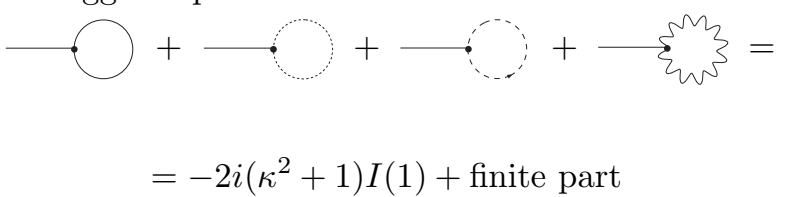

- Higgs propagator

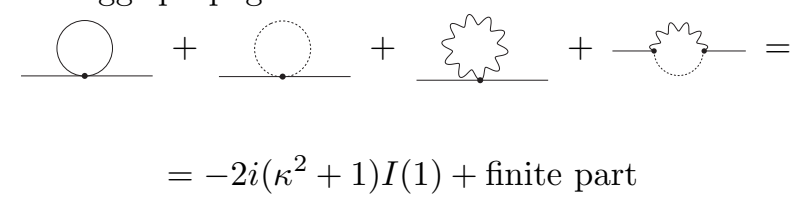

- Goldstone propagator

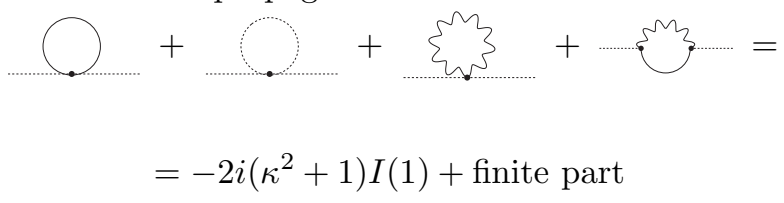

- Vector boson propagator

$$
\begin{aligned}
& \bigcirc^{m}+m+n= \\
& =2 i I(1)+\text { finite part }
\end{aligned}
$$

There are no more one-loop divergent graphs. Therefore, in a minimal subtraction scheme, we add the diagrams shown in the next Table to cancel the divergences in the one-loop graphs.
TABLE III: One-loop counter-terms

\begin{tabular}{cc}
\hline Diagram & Weight \\
\hline & $2 i\left(\kappa^{2}+1\right) I(1)$ \\
$2 i\left(\kappa^{2}+1\right) I(1)$ \\
$2 i\left(\kappa^{2}+1\right) I(1)$ \\
$-2 i I(1)$
\end{tabular}

This is tantamount to considering that the counterterms

$$
\begin{aligned}
\mathcal{L}_{\text {c.t. }}^{S} & =\frac{\hbar\left(\kappa^{2}+1\right)}{2} I(1)\left[|\phi|^{2}-1\right] \\
\mathcal{L}_{\text {c.t. }}^{A} & =-\frac{\hbar}{2} I(1) A_{\mu} A^{\mu}
\end{aligned}
$$

enter into the Lagrangian.

All the finite parts are proportional to $I\left(\kappa^{2}\right)-I(1)$ and they vanish in the critical point between Type I and Type II superconductivity, $\kappa^{2}=1$, to be considered in the sequel. Note that the mass of the elementary particles for this critical value of $\kappa$ is taken as subtraction point so that the counter-terms exactly cancel the divergence due to the Higgs tadpole. Therefore, our renormalization criterion is equivalent to the renormalization condition stipulated in 4] and [5] when $\kappa^{2}=1$.

\section{ANO SELF-DUAL VORTICES}

Abrikosov-Nielsen-Olesen vortices are topological defects satisfying the time-independent field equations:

$$
\partial_{i} F_{i j}=J_{j} \quad ; \quad \frac{1}{2} D_{i} D_{i} \phi=\frac{\partial U}{\partial \phi^{*}} \quad,
$$

where $J_{j}=\frac{i}{2}\left(\phi^{*} D_{j} \phi-\left(D_{j} \phi\right)^{*} \phi\right)$ is the electric current. They are static and localized solutions for which the energy

$$
E=\int d^{2} x\left[\frac{1}{4} F_{i j} F_{i j}+\frac{1}{2}\left(D_{i} \phi\right)^{*} D_{i} \phi+\frac{\kappa}{8}\left(\phi^{*} \phi-1\right)^{2}\right]
$$

is finite. Thus, ANO vortices comply with the boundary conditions on $S_{\infty}^{1}$, i.e. when $r=\sqrt{x_{1}^{2}+x_{2}^{2}}$ tends to $\infty$ :

$$
\left.\phi^{*} \phi\right|_{S_{\infty}^{1}}=1,\left.D_{i} \phi\right|_{S_{\infty}^{1}}=\left.\left(\partial_{i} \phi-i A_{i} \phi\right)\right|_{S_{\infty}^{1}}=0
$$

i.e., $\left.\phi\right|_{S_{\infty}^{1}}=e^{i l \theta}, l \in \mathbb{Z}$, and $\left.A_{i}\right|_{S_{\infty}^{1}}=-\left.i \phi^{*} \partial_{i} \phi\right|_{S_{\infty}^{1}}$. 


\section{First-order equations}

For the value of the coupling constant $\kappa^{2}=1$, the energy functional can be arranged as follows

$E=\int \frac{d^{2} x}{2}\left(\left|D_{1} \phi \pm i D_{2} \phi\right|^{2}+\left[F_{12} \pm \frac{1}{2}\left(\phi^{*} \phi-1\right)\right]^{2}\right)+\frac{1}{2}|g|$

where $g=\int d^{2} x F_{12}=2 \pi l$ is the non-dimensional quantized magnetic flux. Solutions satisfying the first-order differential equations

$$
D_{1} \phi \pm i D_{2} \phi=0 \quad ; \quad F_{12} \pm \frac{1}{2}\left(\phi^{*} \phi-1\right)=0
$$

or, equivalently,

$$
\begin{aligned}
& \left(\partial_{1} \phi_{1}+A_{1} \phi_{2}\right) \mp\left(\partial_{2} \phi_{2}-A_{2} \phi_{1}\right)=0 \\
& \pm\left(\partial_{2} \phi_{1}+A_{2} \phi_{2}\right)+\left(\partial_{1} \phi_{2}-A_{1} \phi_{1}\right)=0 \\
& F_{12} \pm \frac{1}{2}\left(\phi_{1}^{2}+\phi_{2}^{2}-1\right)=0
\end{aligned}
$$

also solve the second-order equations (3) and are called ANO self-dual vortices if they also satisfy the boundary conditions (5). In what follows, we shall focus on solutions with positive $l$ : i.e., we shall choose the upper signs in the first-order equations.

\section{Self-dual vortices with cylindrical symmetry}

If $\theta=\arctan \frac{x_{2}}{x_{1}}$ is the polar angle, the ansatz

$$
\begin{array}{rlrl}
\phi_{1}\left(x_{1}, x_{2}\right)=f(r) \cos l \theta & , & \phi_{2}\left(x_{1}, x_{2}\right)=f(r) \sin l \theta \\
A_{1}\left(x_{1}, x_{2}\right)=-l \frac{\alpha(r)}{r} \sin \theta \quad & A_{1}\left(x_{1}, x_{2}\right)=l \frac{\alpha(r)}{r} \cos \theta
\end{array}
$$

plugged into the first-order equations (6] 17 8) leads to:

$$
\frac{1}{r} \frac{d \alpha}{d r}=\mp \frac{1}{2 l}\left(f^{2}-1\right) \quad, \quad \frac{d f}{d r}= \pm \frac{l}{r} f(r)[1-\alpha(r)] .
$$

Regular solutions of (9) with the boundary conditions $\lim _{r \rightarrow \infty} f(r)=1, \lim _{r \rightarrow \infty} \alpha(r)=1$, zeroes of the Higgs and vector fields at the origin, $f(0)=0, \alpha(0)=0$, and integer magnetic flux,

$$
g=-\oint_{r=\infty} d x_{i} A_{i}=-l \oint_{r=\infty} \frac{\left[x_{2} d x_{1}-x_{1} d x_{2}\right]}{r^{2}}=2 \pi l,
$$

exist and can be found by a mixture of analytical and numerical methods.

Following the procedure developed in [17], we obtain numerical solutions for the vortex equations (9). Indeed, this approach gives the vortex solution in three different ranges of the radial coordinate. For small values of $r$, a power series is tested in the first-order differential equations (9), leading to a recurrence relation between the coefficients. Reference [17] also describes the asymptotic behavior of the solutions. Thus, a numerical scheme can be implemented by setting a boundary condition in a non-singular point of (9), which is obtained from the power series for small values of $r$. This numerical method provides us with the behavior of the vortex solutions for intermediate distances by means of an interpolating polynomial which passes through the numerical data.

The results are shown in figure 1, where the field profiles $\alpha(r)$ and $f(r)$, the magnetic field $B(r)=\frac{l}{2 r} \frac{d \alpha}{d r}$ and the energy density

$$
\varepsilon(r)=\frac{1}{4}\left(1-f^{2}(r)\right)^{2}+\frac{l^{2}}{r^{2}}(1-\alpha(r))^{2} f^{2}(r)
$$

are plotted with respect to $r$ for self-dual ANO vortices with $l=1, l=2, l=3$, and $l=4$. A three-dimensional view of the energy density in the plane is also shown in figure 2 for $l=1, l=2, l=3$, and $l=4$ self-dual vortices. Note that the $l=1$ vortex shows a different pattern as compared with flux tubes of several quanta: only in the first case is the energy density maximum at the origin (the center).
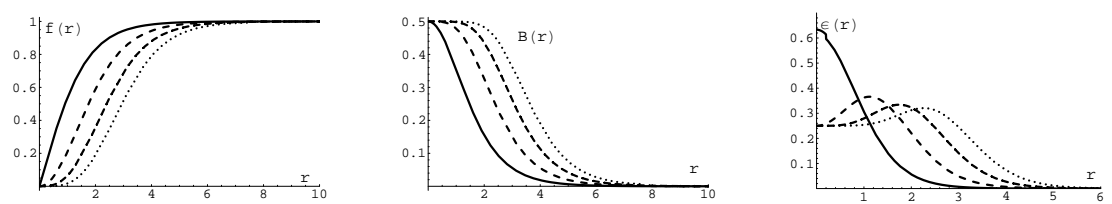

Figure 1. Plots of the field profiles $\alpha(r)$ (a) and $f(r)(b)$, the magnetic field $B(r)(c)$, and the energy density $\varepsilon(r)$ for vortices with $l=1$ (solid line), $l=2$ (broken line), $l=3$ (broken-doted line) and $l=4$ (doted line).
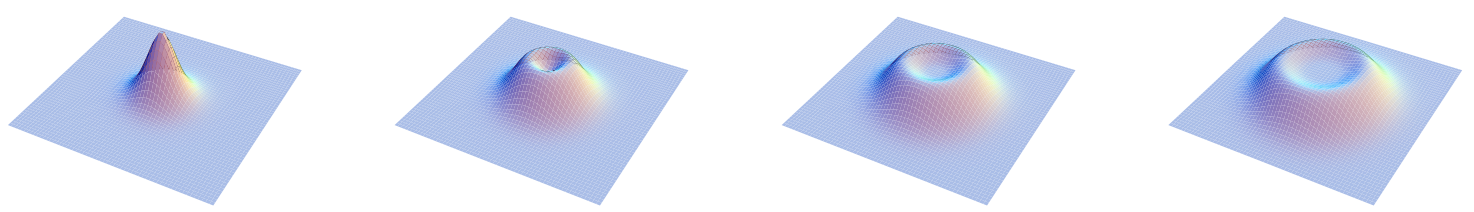

Figure 2. 3D graphics of the energy density for $l=1, l=2, l=3$ and $l=4$ self-dual symmetric ANO vortices. 


\section{Two-vortex solutions with distinct centers}

To tackle the task of building $l=2$ ANO self-dual solutions formed by two $l=1$ vortices with centers separated by a distance $d$, we follow the work [18] by Jacobs and Rebbi. A variational method is implemented in two stages:

In the first stage, trial functions depending only on a single variational parameter $w$ are considered:

$$
\begin{aligned}
\phi_{\omega}\left(z, z^{*}\right) & =\Phi\left(z, z^{*}\right)\left[\omega f^{(1)}(|z-d / 2|) f^{(1)}(|z+d / 2|)+(1-\omega) \frac{\left|z^{2}-(d / 2)^{2}\right|}{\left|z^{2}\right|} f^{(2)}(|z|)\right] \\
A^{\omega}\left(z, z^{*}\right) & =\omega\left(\frac{i}{z^{*}-d / 2} \alpha^{(1)}(|z-d / 2|)+\frac{i}{z^{*}+d / 2} \alpha^{(1)}(|z+d / 2|)\right)+(1-\omega) \frac{2 i}{z^{*}} \alpha^{(2)}(|z|)
\end{aligned}
$$

Here

$z=x_{1}+i x_{2} \quad, \quad A^{\omega}\left(z, z^{*}\right)=A_{1}^{\omega}\left(z, z^{*}\right)+i A_{2}^{\omega}\left(z, z^{*}\right)$

and

$$
\Phi=\sqrt{\frac{z^{2}-(d / 2)^{2}}{z^{* 2}-(d / 2)^{2}}}
$$

is essentially a phase chosen in such a way that the magnetic flux is equal to $4 \pi . f^{(1)}, \alpha^{(1)}, f^{(2)}$ and $\alpha^{(2)}$ stand for the functions $f$ and $\alpha$ associated with self-dual solutions with cylindrical symmetry -obtained in the previous subsection- respectively with vorticity $l=1$ and $l=2$. Evoking (10) and (11) we expect that $\omega=0$ for the case ,$d=0$ and $\omega=1$ for the case $d>>1$. Plugging (10) and (11) into the energy functional, we obtain a expression $E(\omega)$, which is set to be minimized as a function of $\omega$.

In the second stage the trial functions are refined by adding a deformation such that two requirements are fulfilled: 1) the scalar field vanishes at the two centers. 2) the gauge-invariant quantities associated with the solution are symmetric with respect to the reflection $z \rightarrow z^{*}$. The invariant ansatz reads:

$$
\begin{aligned}
& \phi\left(z, z^{*}\right)=\phi_{\omega}\left(z, z^{*}\right)+\Phi\left(z, z^{*}\right)\left|z^{2}-(d / 2)^{2}\right|(\cosh |z|)^{-1} \sum_{i=0}^{N} \sum_{j=0}^{i} f_{i j} \frac{\left(z z^{*}\right)^{i}}{2}\left[\left(\frac{z}{z^{*}}\right)^{j}+\left(\frac{z^{*}}{z}\right)^{j}\right] \\
& A\left(z, z^{*}\right)=A^{\omega}\left(z, z^{*}\right)+\frac{1}{\cosh |z|}\left\{z \sum_{i=0}^{N} \sum_{j=0}^{i} a_{i j}^{I} \frac{\left(z z^{*}\right)^{i}}{2}\left[\left(\frac{z}{z^{*}}\right)^{j}+\left(\frac{z^{*}}{z}\right)^{j}\right]+z^{*} \sum_{i=0}^{N} \sum_{j=0}^{i} a_{i j}^{I I} \frac{\left(z z^{*}\right)^{i}}{2}\left[\left(\frac{z}{z^{*}}\right)^{j}+\left(\frac{z^{*}}{z}\right)^{j}\right]\right\}
\end{aligned}
$$
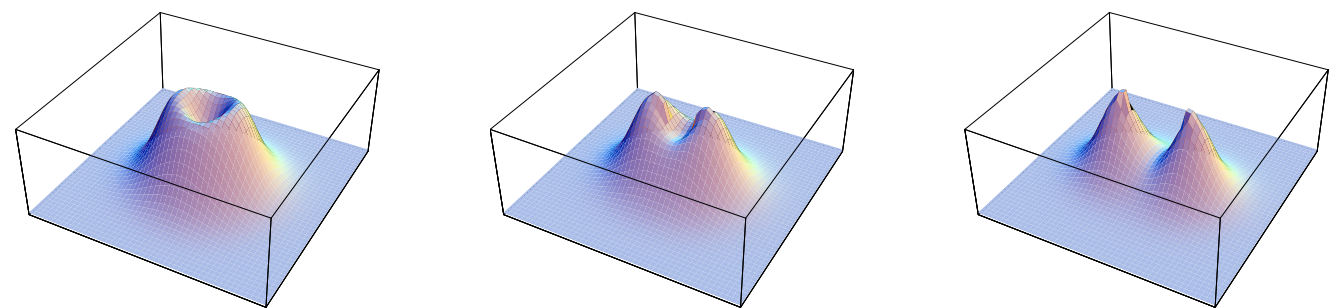

Figure 3. $3 D$ graphics of the energy density for $l=2$ self-dual separate vortices with centers at distances $d=1, d=2, d=3$.

These expressions involve $\aleph=3 \frac{(N+1)(N+2)}{2}$ variational parameters $f_{i j}, a_{i j}^{I}, a_{i j}^{I I}$. Finding the minimum of the energy functional as a function of these $\aleph$ variables - a task for Mathematica- a good approximation to the $l=2$ self-dual solution with a distance $d$ between the two $l=$
1 vortex centers is obtained. For our purposes setting $N=1$ such that $\aleph=9$ will suffice. The energy density for two-vortex solutions found by this method if $\aleph=9$ is depicted for $d=1, d=2$ and $d=3$ in the above figure. 


\section{Small fluctuations}

We generically denote the vortex solution fields as $\phi^{V}=\psi=\psi_{1}+i \psi_{2} \quad, \quad A_{k}^{V}=V_{k} \quad, k=1,2 \quad$.

Assembling the small fluctuations around the solution

$$
\phi(\vec{x})=\psi(\vec{x})+\varphi(\vec{x}) \quad, \quad A_{k}(\vec{x})=V_{k}(\vec{x})+a_{k}(\vec{x})
$$

in a four column $\xi(\vec{x}), \mathrm{L}^{2}$-integrable second-order fluctuations around a given vortex solution are still solutions of the first-order equations with the same magnetic flux if they belong to the kernel of the Dirac-like operator, $\mathcal{D} \xi(\vec{x})=0,19$

$$
\mathcal{D} \xi(\vec{x})=\left(\begin{array}{cccc}
-\partial_{2} & \partial_{1} & \psi_{1} & \psi_{2} \\
-\partial_{1} & -\partial_{2} & -\psi_{2} & \psi_{1} \\
\psi_{1} & -\psi_{2} & -\partial_{2}+V_{1} & -\partial_{1}-V_{2} \\
\psi_{2} & \psi_{1} & \partial_{1}+V_{2} & -\partial_{2}+V_{1}
\end{array}\right)\left(\begin{array}{c}
a_{1}(\vec{x}) \\
a_{2}(\vec{x}) \\
\varphi_{1}(\vec{x}) \\
\varphi_{2}(\vec{x})
\end{array}\right)
$$

The first component of $\mathcal{D} \xi$ gives the deformation of the vortex equation (8), whereas the third and fourth components are due to the respective deformation of the covariant holomorphy equations (7) and (6). The second component sets the background gauge

$$
B\left(a_{k}, \varphi ; \psi\right)=\partial_{k} a_{k}-\left(\psi_{1} \varphi_{2}-\psi_{2} \varphi_{1}\right)
$$

on the fluctuations.

The operator $\mathcal{H}^{+}=\mathcal{D}^{\dagger} \mathcal{D}$ and its partner $\mathcal{H}^{-}=\mathcal{D} \mathcal{D}^{\dagger}$ read:

$$
\begin{aligned}
& \mathcal{H}^{+}=\left(\begin{array}{cccc}
-\triangle+|\psi|^{2} & 0 & -2 \nabla_{1} \psi_{2} & 2 \nabla_{1} \psi_{1} \\
0 & -\triangle+|\psi|^{2} & -2 \nabla_{2} \psi_{2} & 2 \nabla_{2} \psi_{1} \\
-2 \nabla_{1} \psi_{2} & -2 \nabla_{2} \psi_{2} & -\triangle+\frac{1}{2}\left(3|\psi|^{2}+2 V_{k} V_{k}-1\right) & -2 V_{k} \partial_{k} \\
2 \nabla_{1} \psi_{1} & 2 \nabla_{2} \psi_{1} & 2 V_{k} \partial_{k} & -\triangle+\frac{1}{2}\left(3|\psi|^{2}+2 V_{k} V_{k}-1\right)
\end{array}\right) \\
& \mathcal{H}^{-}=\left(\begin{array}{cccc}
-\triangle+|\psi|^{2} & 0 & 0 & 0 \\
0 & -\triangle+|\psi|^{2} & 0 & 0 \\
0 & 0 & -\triangle+\frac{1}{2}\left(|\psi|^{2}+1\right)+V_{k} V_{k} & -2 V_{k} \partial_{k} \\
0 & 0 & 2 V_{k} \partial_{k} & -\triangle+\frac{1}{2}\left(|\psi|^{2}+1\right)+V_{k} V_{k}
\end{array}\right) .
\end{aligned}
$$

One can check that $\mathcal{H}^{+}$arises in the small deformation of the second-order equations (3) in the background gauge for $\kappa=1$, thus ruling the second-order fluctuations around the vortex solutions. In fact, for $l=0$ one finds that $\mathcal{H}^{+}=\mathcal{H}^{-}=\mathcal{H}_{0}$, where

$$
\mathcal{H}_{0}=\left(\begin{array}{cccc}
-\triangle+1 & 0 & 0 & 0 \\
0 & -\triangle+1 & 0 & 0 \\
0 & 0 & -\triangle+1 & 0 \\
0 & 0 & 0 & -\Delta+1
\end{array}\right)
$$

is the second-order fluctuation operator around the vacuum in the Feynman-'t Hooft renormalizable gauge: the background gauge in the vacuum sector. Note that the fluctuations in this gauge correspond to a massive vector particle plus scalar Higgs and Goldstone fields. It will be useful in the sequel to write the second-order fluctuation operators around $l \geq 1$ vortices in the form:

$$
\mathcal{H}^{ \pm}=\mathcal{H}_{0}+Q_{k}^{ \pm}(\vec{x}) \partial_{k}+V^{ \pm}(\vec{x}) \quad
$$

where $Q_{k}^{ \pm}(\vec{x})$ and $V^{ \pm}(\vec{x})$ are $4 \times 4$ functional matrices. 


\section{HIGH-TEMPERATURE EXPANSION OF HEAT TRACES}

\section{Index theorem: moduli space of self-dual vortices}

One easily checks that $\operatorname{dim} \operatorname{ker} \mathcal{D}^{\dagger}=0$ because the spectrum of $\mathcal{H}^{-}$is definite positive. Thus, the dimension of the moduli space of self-dual vortex solutions with magnetic charge $l$ is the index of $\mathcal{D}$ :

$$
\text { ind } \mathcal{D}=\operatorname{dim} \operatorname{ker} \mathcal{D}-\operatorname{dim} \operatorname{ker} \mathcal{D}^{\dagger}
$$

We follow Weinberg 19, using the background instead of the Coulomb gauge, to briefly determine ind $\mathcal{D}$. The spectra of the operators $\mathcal{H}^{+}$and $\mathcal{H}^{-}$only differ in the number of eigen-functions belonging to their kernels. For topological vortices, we do not expect pathologies due to asymmetries between the spectral densities of $\mathcal{H}^{+}$and $\mathcal{H}^{-}$, and thus ind $\mathcal{D}=\operatorname{Tr} e^{-\beta \mathcal{H}^{+}}-\operatorname{Tr} e^{-\beta \mathcal{H}^{-}}$. For a case in which these asymmetries are important, see the treatment of Chern-Simons-Higgs topological and nontopological vortices given in 20, 21.

The heat trace of a $N \times N$ matrix differential operator

$$
\mathcal{H}=\mathcal{H}_{0}+Q_{k}(\vec{x}) \partial_{k}+V(\vec{x})
$$

-like the $\mathcal{H}^{ \pm}$operators- is defined as

$$
\operatorname{Tr} e^{-\beta \mathcal{H}}=\operatorname{tr} \int_{\mathbb{R}^{2}} d^{2} \vec{x} K_{\mathcal{H}}(\vec{x}, \vec{x} ; \beta)
$$

where $K_{\mathcal{H}}(\vec{x}, \vec{y} ; \beta)$ is the $N \times N$ matrix kernel of the heat equation and $\operatorname{tr}$ is the usual matrix trace. Therefore, $K_{\mathcal{H}}(\vec{x}, \vec{y} ; \beta)$ solves the heat equation

$$
\left(\frac{\partial}{\partial \beta} \mathbb{I}+\mathcal{H}\right) K_{\mathcal{H}}(\vec{x}, \vec{y} ; \beta)=0
$$

with initial condition

$$
K_{\mathcal{H}}(\vec{x}, \vec{y} ; 0)=\mathbb{I} \cdot \delta^{(2)}(\vec{x}-\vec{y})
$$

Because

$$
K_{\mathcal{H}_{0}}(\vec{x}, \vec{y} ; \beta)=\frac{e^{-\beta}}{4 \pi \beta} \cdot \mathbb{I} \cdot e^{-\frac{|\vec{x}-\vec{y}|}{4 \beta}}
$$

is the heat kernel for the Klein-Gordon operator $\mathcal{H}_{0}$, it is convenient to write the heat kernel for $\mathcal{H}$ in the form:

$$
K_{\mathcal{H}}(\vec{x}, \vec{y} ; \beta)=C_{\mathcal{H}}(\vec{x}, \vec{y} ; \beta) K_{\mathcal{H}_{0}}(\vec{x}, \vec{y} ; \beta)
$$

with $C_{\mathcal{H}}(\vec{x}, \vec{x} ; 0)=\mathbb{I}$ 23]. Substituting (14) into (12) we find that $C_{\mathcal{H}}(\vec{x}, \vec{y} ; \beta)$ solves the transfer equations:

$$
\begin{aligned}
\left\{\frac{\partial}{\partial \beta} \mathbb{I}+\right. & \frac{x_{k}-y_{k}}{\beta}\left(\partial_{k} \mathbb{I}-\frac{1}{2} Q_{k}\right)-\triangle \mathbb{I}+ \\
& \left.+Q_{k} \partial_{k}+V\right\} C_{\mathcal{H}}(\vec{x}, \vec{y} ; \beta)=0
\end{aligned}
$$

The high-temperature expansion

$$
C_{\mathcal{H}}(\vec{x}, \vec{y} ; \beta)=\sum_{n=0}^{\infty} c_{n}(\vec{x}, \vec{y} ; \mathcal{H}) \beta^{n}
$$

trades the PDE (15) by the recurrence relations

$$
\begin{array}{r}
{\left[n \mathbb{I}+\left(x_{k}-y_{k}\right)\left(\partial_{k} \mathbb{I}-\frac{1}{2} Q_{k}\right)\right] c_{n}(\vec{x}, \vec{y} ; \mathcal{H})=} \\
=\left[\triangle \mathbb{I}-Q_{k} \partial_{k}-V\right] c_{n-1}(\vec{x}, \vec{y} ; \mathcal{H})
\end{array}
$$

among the local coefficients with $n \geq 1$, with the initial condition $c_{0}(\vec{x}, \vec{x} ; \mathcal{H})=\mathbb{I}$. Taking into account that

$$
\begin{aligned}
\operatorname{Tr} e^{-\beta \mathcal{H}} & =\frac{e^{-\beta}}{4 \pi \beta} \sum_{n=0}^{\infty} \sum_{a=1}^{4} \int d^{2} x\left[c_{n}\right]_{a a}(\vec{x}, \vec{x} ; \mathcal{H}) \beta^{n}= \\
& =\frac{e^{-\beta}}{4 \pi \beta} \sum_{n=0}^{\infty} \beta^{n} c_{n}(\mathcal{H})
\end{aligned}
$$

where we have defined the Seeley coefficients as

$$
c_{n}(\mathcal{H})=\sum_{a=1}^{4} \int d^{2} x\left[c_{n}\right]_{a a}(\vec{x}, \vec{x} ; \mathcal{H}) \quad,
$$

and that the first local coefficient can be easily computed

$$
c_{1}(\vec{x}, \vec{x} ; \mathcal{H})=-V(\vec{x}) \quad,
$$

by applying these formulas to the $\mathcal{H}^{ \pm}$operators we obtain in the $\beta=0$-infinite temperature- limit :

$$
\begin{aligned}
\operatorname{ind} \mathcal{D} & =\frac{1}{4 \pi}\left\{c_{1}\left(\mathcal{H}^{+}\right)-c_{1}\left(\mathcal{H}^{-}\right)\right\}= \\
& =\frac{1}{\pi} \int d^{2} x\left(\frac{\partial V_{2}}{\partial x_{1}}-\frac{\partial V_{1}}{\partial x_{2}}\right)(\vec{x})=2 l,
\end{aligned}
$$

i.e., the dimension of the self-dual vortex moduli space is $2 l$. Physically, this means that there are solutions, if $\kappa=1$, for any location of the $l$-vortex centers in the plane [24]; all static configurations of self-dual $l$-vortices can thus be interpreted as states of neutral equilibrium.

\section{Seeley coefficients}

Computation of the coefficients of the asymptotic expansion is a difficult task; to start with, the order two local coefficient reads:

$$
\begin{aligned}
& c_{2}(\vec{x}, \vec{x} ; \mathcal{H})=-\frac{1}{6} \triangle V(\vec{x})+\frac{1}{12} Q_{k}(\vec{x}) Q_{k}(\vec{x}) V(\vec{x})- \\
& -\frac{1}{6} \partial_{k} Q_{k}(\vec{x}) V(\vec{x})+\frac{1}{6} Q_{k}(\vec{x}) \partial_{k} V(\vec{x})+\frac{1}{2} V^{2}(\vec{x}) .
\end{aligned}
$$

Complexity increases strongly for high-order local coefficients .

The recurrence relation (16) allows us to express $c_{n}(\vec{x}, \vec{y}, \mathcal{H})$ and its derivatives in terms of all the 
$c_{k}(\vec{x}, \vec{y} ; \mathcal{H})$ with $k \leq n$ and their derivatives. One passes from this information to the values of the Seeley coefficients $c_{n}(\mathcal{H})$ in two steps. First, one must reach the subtle $\vec{y} \rightarrow \vec{x}$ limit. In this analytical manoeuvre the partial derivatives of $c_{n}(\vec{x}, \vec{y} ; \mathcal{H})$ at $\vec{y}=\vec{x}$

$$
{ }^{\left(\alpha_{1}, \alpha_{2}\right)} C_{n}^{a b}(\vec{x})=\lim _{\vec{y} \rightarrow \vec{x}} \frac{\partial^{\alpha_{1}+\alpha_{2}}\left[c_{n}\right]_{a b}(\vec{x}, \vec{y} ; \mathcal{H})}{\partial x_{1}^{\alpha_{1}} \partial x_{2}^{\alpha_{2}}}
$$

play a prominent rôle. Note also that:

$$
\left[c_{n}\right]_{a b}(\vec{x}, \vec{x} ; \mathcal{H})={ }^{(0,0)} C_{n}^{a b}(\vec{x})
$$

In the $\vec{y} \rightarrow \vec{x}$ limit the recurrence relation (16) becomes :

$$
\begin{aligned}
\left(k+\alpha_{1}+\right. & \left.\alpha_{2}+1\right)^{\left(\alpha_{1}, \alpha_{2}\right)} C_{k+1}^{a b}(\vec{x})={ }^{\left(\alpha_{1}+2, \alpha_{2}\right)} C_{k}^{a b}(\vec{x})+{ }^{\left(\alpha_{1}, \alpha_{2}+2\right)} C_{k}^{a b}(\vec{x})- \\
& -\sum_{d=1}^{N} \sum_{r=0}^{\alpha_{1}} \sum_{t=0}^{\alpha_{2}}\left(\begin{array}{c}
\alpha_{1} \\
r
\end{array}\right)\left(\begin{array}{c}
\alpha_{2} \\
t
\end{array}\right)\left[\frac{\partial^{r+t} Q_{1}^{a d}}{\partial x_{1}^{r} \partial x_{2}^{t}}{ }^{\left(\alpha_{1}-r+1, \alpha_{2}-t\right)} C_{k}^{d b}(\vec{x})+{\left.\frac{\partial^{r+t} Q_{2}^{a d}}{\partial x_{1}^{r} \partial x_{2}^{t}}{ }^{\left(\alpha_{1}-r, \alpha_{2}-t+1\right)} C_{k}^{d b}(\vec{x})\right]+}+\frac{1}{2} \sum_{d=1}^{N} \sum_{r=0}^{\alpha_{1}-1} \sum_{t=0}^{\alpha_{2}} \alpha_{1}\left(\begin{array}{c}
\alpha_{1}-1 \\
r
\end{array}\right)\left(\begin{array}{c}
\alpha_{2} \\
t
\end{array}\right) \frac{\partial^{r+t} Q_{1}^{a d}}{\partial x_{1}^{r} \partial x_{2}^{t}}{ }^{\left(\alpha_{1}-1-r, \alpha_{2}-t\right)} C_{k+1}^{d b}(\vec{x})+\right. \\
& +\frac{1}{2} \sum_{d=1}^{N} \sum_{r=0}^{\alpha_{2}-1} \sum_{t=0}^{\alpha_{1}} \alpha_{2}\left(\begin{array}{c}
\alpha_{2}-1 \\
r
\end{array}\right)\left(\begin{array}{c}
\alpha_{1} \\
t
\end{array}\right){\frac{\partial^{r+t} Q_{2}^{a d}}{\partial x_{1}^{t} \partial x_{2}^{r}}\left(\alpha_{1}-t, \alpha_{2}-1-r\right) C_{k+1}^{d b}(\vec{x})-}-\sum_{d=1}^{N} \sum_{r=0}^{\alpha_{2}} \sum_{t=0}^{\alpha_{1}}\left(\begin{array}{c}
\alpha_{1} \\
t
\end{array}\right)\left(\begin{array}{c}
\alpha_{2} \\
r
\end{array}\right) \frac{\partial^{r+t} V^{a d}}{\partial x_{1}^{t} \partial x_{2}^{r}}\left(\alpha_{1}-t, \alpha_{2}-r\right) C_{k}^{d b}(\vec{x})
\end{aligned}
$$

The initial condition $c_{0}(\vec{x}, \vec{x} ; \mathcal{H})=\mathbb{I}$ means that all the ${ }^{(\beta, \gamma)} C_{0}^{a b}(\vec{x})$ vanish except ${ }^{(0,0)} C_{0}^{a a}(\vec{x})=1$ for $a=$ $1,2, \cdots, N$. Starting from these conditions one computes all the ${ }^{(\beta, \gamma)} C_{n}^{a b}(\vec{x})$ local coefficients by using (18). For instance, in order to obtain ${ }^{(0,0)} C_{6}^{a b}(\vec{x})$ for $\mathcal{H}^{+}$we need ${ }^{(\beta, \gamma)} C_{5}^{a b}(\vec{x})$ for $\beta, \gamma=0,1,2$ as data, which in turn can be calculated from ${ }^{(\beta, \gamma)} C_{4}^{a b}(\vec{x})$ for $\beta, \gamma=0,1,2,3,4$, and so forth. Evaluation of ${ }^{(0,0)} C_{6}^{a b}(\vec{x})$ requires knowledge of 4032 local coefficients !!!. In general, the rule is: knowledge of ${ }^{(0,0)} C_{n}^{a b}(\vec{x})$ amounts to knowledge of $\frac{8}{3}(n+1)(n+2)(4 n+3){ }^{(\beta, \gamma)} C_{k}^{a b}(\vec{x})$ local coefficients with $k \leq n$. The second step is much simpler: simple numerical integration of $\sum_{a=1}^{4}{ }^{(0,0)} C_{n}^{a a}(\vec{x})$ over the plane.

\section{QUANTUM OSCILLATIONS OF SELF-DUAL VORTICES}

Standard lore in the semi-classical quantization of solitons tells us that the one-loop mass shift comes from the Casimir energy plus the contribution of the mass renormalization counter-terms: $\Delta M_{V}=\Delta M_{V}^{C}+\Delta M_{V}^{R}$.

\section{Casimir energy and vortex mass renormalization counter-terms}

By expanding the static energy (4) of the AHM around self-dual vortex solutions one obtains, up to second-order in $\xi$ in the background gauge:

$$
\begin{aligned}
E & +\frac{v^{2}}{2} \int d^{2} \vec{x}\left[\partial_{j} a_{j}-\psi_{1} \varphi_{2}+\psi_{2} \varphi_{1}\right]^{2} \\
& \simeq \pi|l| v^{2}+\frac{1}{2} \int d^{2} \vec{x} \xi^{T} \mathcal{H}^{+} \xi+\mathcal{O}\left(\xi^{3}\right)
\end{aligned}
$$

Also, the ghosts -arising when the quantization procedure is performed in the background gauge- contribute negatively to the energy:

$$
E^{\mathrm{Ghost}}=\frac{v^{2}}{2} \int d^{2} \vec{x}\left[\chi^{*}\left(-\triangle+|\psi|^{2}\right) \chi+\psi^{*} \varphi \chi^{*} \chi\right] .
$$

Thus, the vortex Casimir energy is the sum of the Casimir energies of the bosonic $a_{1}, a_{2}, \varphi_{1}, \varphi_{2}$ fluctuations around the vortex minus the Casimir energy of the fermionic fluctuation $\chi$; the ordinary -non-matrix- Schrodinger operator ruling the ghost fluctuation around the vortex is:

$$
\mathcal{H}^{G}=-\triangle+|\psi|^{2} \quad .
$$

$\varphi_{2}$ is a pure gauge oscillation but its contribution is killed by the negative ghost contribution. The same applies for the vacuum Casimir energy: the Goldstone boson Casimir energy is canceled by the ghost Casimir energy, the trace of the square root of $\mathcal{H}_{0}^{G}=-\Delta+1$. In sum, the vortex Casimir energy measured with respect to the vacuum Casimir energy is given by the formal formula:

$$
\Delta M_{V}^{C}=\frac{\hbar m}{2}\left[\operatorname{STr}^{*}\left(\mathcal{H}^{+}\right)^{\frac{1}{2}}-\mathrm{S} \operatorname{Tr}\left(\mathcal{H}_{0}\right)^{\frac{1}{2}}\right]
$$




$$
\begin{aligned}
\operatorname{STr}^{*}\left(\mathcal{H}^{+}\right)^{\frac{1}{2}} & =\operatorname{Tr}^{*}\left(\mathcal{H}^{+}\right)^{\frac{1}{2}}-\operatorname{Tr}\left(\mathcal{H}^{G}\right)^{\frac{1}{2}} \\
\operatorname{STr}\left(\mathcal{H}_{0}\right)^{\frac{1}{2}} & =\operatorname{Tr}\left(\mathcal{H}_{0}\right)^{\frac{1}{2}}-\operatorname{Tr}\left(\mathcal{H}_{0}^{G}\right)
\end{aligned}
$$

The star means that the $2 l$ zero eigenvalues of $\mathcal{H}^{+}$must be subtracted because zero modes only enter at two-loop order.

In the minimal subtraction renormalization scheme, finite renormalizations are adjusted in such a way that the critical point $\kappa^{2}=1$ is reached at first-order in the loop expansion. Therefore, (11) and (2) tell us that the contribution of the mass renormalization counter-terms to the vortex mass is:

$$
\begin{aligned}
\Delta M_{V}^{R} & =\Delta M_{c . t .}^{S}+\Delta M_{c . t .}^{A}=\hbar m I(1) \Sigma\left(\psi, V_{k}\right) \\
\Sigma\left(\psi, V_{k}\right) & =\int d x^{2}\left[\left(1-|\psi|^{2}\right)-\frac{1}{2} V_{k} V_{k}\right],
\end{aligned}
$$

and the divergent integral $I(1)$ can be written in the form

$$
I(1)=\frac{1}{2} \int \frac{d^{2} \vec{k}}{(2 \pi)^{2}} \frac{1}{\sqrt{\vec{k} \cdot \vec{k}+1}}
$$

after applying the residue theorem to integration in the complex $k_{0}$-plane.

\section{Zeta function regularization of Casimir energies and self-energy graphs}

We regularize both infinite quantities $\Delta M_{V}^{C}$ and $\Delta M_{V}^{R}$ by means of generalized zeta functions. From the spectral resolution of a Fredholm operator $\mathcal{H}$

$$
\mathcal{H} \xi_{n}=\lambda_{n} \xi_{n} \quad,
$$

one defines the generalized zeta function as the series

$$
\zeta_{\mathcal{H}}(s)=\sum_{n} \frac{1}{\lambda_{n}^{s}}
$$

which is a meromorphic function of the complex variable $s$ [22], 23]. We can then hope that, despite their continuous spectra, our operators fit in this scheme, and write:

$$
\begin{aligned}
\Delta M_{V}^{C}(s)= & \frac{\hbar \mu}{2}\left(\frac{\mu^{2}}{m^{2}}\right)^{s}\left\{\left(\zeta_{\mathcal{H}^{+}}(s)-\zeta_{\mathcal{H}^{G}}(s)\right)+\right. \\
& \left.+\left(\zeta_{\mathcal{H}_{0}^{G}}(s)-\zeta_{\mathcal{H}_{0}}(s)\right)\right\} \\
\Delta M_{V}^{R}(s)= & \frac{\hbar}{m L^{2}} \zeta_{\mathcal{H}_{0}}(s) \Sigma\left(\psi, V_{k}\right)
\end{aligned}
$$

where

$$
\zeta_{\mathcal{H}_{0}}(s)=\frac{m^{2} L^{2}}{4 \pi} \frac{\Gamma(s-1)}{\Gamma(s)}
$$

and $\mu$ is a parameter of inverse length dimensions. Note that

$$
\Delta M_{V}^{C}=\lim _{s \rightarrow-\frac{1}{2}} \Delta M_{V}^{C}(s) \quad, \quad \Delta M_{V}^{R}=\lim _{s \rightarrow \frac{1}{2}} \Delta M_{V}^{R}(s)
$$

and

$$
I(1)=\lim _{s \rightarrow \frac{1}{2}} \frac{1}{2 m^{2} L^{2}} \zeta_{\mathcal{H}_{0}}(s)
$$

on a square of area $L^{2}$.

Together with the high-temperature expansion, the Mellin transform of the heat trace

$$
\zeta_{\mathcal{H}}(s)=\frac{1}{\Gamma(s)} \int_{0}^{\infty} d \beta \beta^{s-1} \operatorname{Tr} e^{-\beta \mathcal{H}}
$$

shows that

$$
\zeta_{\mathcal{H}}(s)=\frac{1}{\Gamma(s)} \sum_{n=0}^{\infty} \int_{0}^{1} d \beta \beta^{s+n-2} c_{n}(\mathcal{H}) e^{-\beta}+\frac{1}{\Gamma(s)} B_{\mathcal{H}}(s)
$$

is the sum of meromorphic and entire $-B_{\mathcal{H}}(s)$ - functions of $s$. Neglecting the entire parts and keeping a finite number of terms, $N_{0}$, in the asymptotic series for $\zeta_{\mathcal{H}}(s)$, we find the following approximations for the generalized zeta functions concerning the differential operators $\mathcal{H}^{+}$ and $\mathcal{H}^{G}$ relevant to our problem:

$$
\begin{aligned}
\zeta_{\mathcal{H}^{+}}(s)-\zeta_{\mathcal{H}_{0}}(s) & \simeq \sum_{n=1}^{N_{0}} c_{n}\left(\mathcal{H}^{+}\right) \cdot \frac{\gamma[s+n-1,1]}{4 \pi \Gamma(s)} \\
\zeta_{\mathcal{H}_{0}^{G}}(s)-\zeta_{\mathcal{H}^{G}}(s) & \simeq-\sum_{n=1}^{N_{0}} c_{n}\left(\mathcal{H}^{G}\right) \cdot \frac{\gamma[s+n-1,1]}{4 \pi \Gamma(s)} ;
\end{aligned}
$$

$\gamma[s+n-1,1]=\int_{0}^{1} d \beta \beta^{s+n-2} e^{-\beta}$ is the incomplete gamma function, with a very well known meromorphic structure.

Regarding one-dimensional kinks, see 9], 12], 13], the contributions of $c_{0}\left(\mathcal{H}^{+}\right)$and $c_{0}\left(\mathcal{H}^{G}\right)$ to $\zeta_{\mathcal{H}^{+}}(s)$ and $\zeta_{\mathcal{H}^{G}}(s)$ are respectively canceled by $\zeta_{\mathcal{H}_{0}}(s)$ and $\zeta_{\mathcal{H}_{0}^{G}}(s)$; i.e., renormalization of zero point vacuum energies takes care of the $c_{0}\left(\mathcal{H}^{+}\right)$and $c_{0}\left(\mathcal{H}^{G}\right)$ contributions to the vortex Casimir energy. Note, however, that, in contrast to the $(1+1)$-dimensional case, the value $s=-\frac{1}{2}$ for which we shall obtain the Casimir energy is not a pole. To compute the vortex Casimir energy one can first take the $s=-\frac{1}{2}$ limit and then subtract the vacuum Casimir energy regularized by this procedure; a finite answer for the kink Casimir energy is only reached if one first subtracts the vacuum Casimir energy of the one-dimensional system.

\section{One-loop mass shift formula}

Writing as $\bar{c}_{n}=c_{n}\left(\mathcal{H}^{+}\right)-c_{n}\left(\mathcal{H}^{G}\right)$ the difference between the Seeley coefficients of $\mathcal{H}^{+}$and $\mathcal{H}^{G}$ for vorticity $l$, we check that the contribution of the first coefficient to the Casimir energy

$$
\Delta M_{V}^{(1) C}(s) \simeq \frac{\hbar}{2} \mu\left(\frac{\mu^{2}}{m^{2}}\right)^{s} \bar{c}_{1} \cdot \frac{\gamma[s, 1 / 2]}{4 \pi \Gamma(s)}
$$


is finite at the $s \rightarrow-\frac{1}{2}$ limit

$$
\Delta M_{V}^{(1) C}(-1 / 2) \simeq-\frac{\hbar m}{4 \pi} \Sigma\left(\psi, V_{k}\right) \cdot \frac{\gamma[-1 / 2,1]}{\Gamma(1 / 2)}
$$

and exactly cancels the contribution of the mass renormalization counter-terms -also finite for $s=\frac{1}{2}-$ :

$$
\begin{aligned}
\Delta M_{V}^{R}(s) & \simeq \frac{\hbar m}{4 \pi} \cdot \Sigma\left(\psi, V_{k}\right) \cdot \frac{\gamma[s-1,1]}{\Gamma(s)} \\
\Delta M_{V}^{R}(1 / 2) & \simeq \frac{\hbar m}{4 \pi} \cdot \Sigma\left(\psi, V_{k}\right) \cdot \frac{\gamma[-1 / 2,1]}{\Gamma(1 / 2)}
\end{aligned}
$$

Our choice of a minimal subtraction scheme not only arranges finite renormalizations in such a way that selfduality holds for $\kappa=1$ at the one-loop order, but also fits in with the criterion that the mass renormalization counter-terms must kill the contribution to the Casimir energy of the first Seeley coefficients for the heat trace expansions of the operators $\mathcal{H}^{+}, \mathcal{H}^{G}, \mathcal{H}_{0}, \mathcal{H}_{0}^{G}$. The same cancellation happens for kinks only if the mode number cut-off regularization procedure, see [6], 9] and 25], is applied.

Subtracting the contribution of the $2 l$ zero modes,

$$
\begin{aligned}
\Delta M_{V}= & \frac{\hbar m}{2} \lim _{s \rightarrow-\frac{1}{2}}\left[-\frac{2 l}{\Gamma(s)} \int_{0}^{1} d \beta \beta^{s-1}+\right. \\
& \left.+\sum_{n=2}^{N_{0}} \bar{c}_{n} \frac{\gamma[s+n-1,1]}{4 \pi \Gamma(s)}\right]
\end{aligned}
$$

we finally obtain the following formula for the vortex mass shift:

$$
\Delta M_{V}=-\frac{\hbar m}{2}\left[\frac{1}{8 \pi \sqrt{\pi}} \sum_{n=2}^{N_{0}} \bar{c}_{n} \gamma\left[n-\frac{3}{2}, 1\right]+\frac{2 l}{\sqrt{\pi}}\right] .
$$

\section{ONE-LOOP MASS SHIFTS}

\section{Local coefficients for cylindrically symmetric vortices}

We shall apply these formulae to cylindrically symmetric vortices. The heat kernel local coefficients, how- ever, depend on successive derivatives of the solution. This dependence can increase the error in the estimation of these local coefficients because we handle an interpolating polynomial as the numerically generated solution, and the successive derivations with respect to $r$ of such a polynomial introduces inaccuracies. Indeed this operation is plugged into the algorithm that generates the local coefficients in order to speed up this process. It is thus of crucial importance to use the first-order differential equations (9) in order to eliminate the derivatives of the solution and write the local coefficients as expressions depending only on the fields. We find:

$$
\begin{aligned}
& \frac{\partial \psi_{1}}{\partial x_{1}}=\frac{l f(r)}{r}[\cos \theta \cos l \theta(1-\alpha(r))+\sin \theta \sin l \theta] \\
& \frac{\partial \psi_{1}}{\partial x_{2}}=\frac{l f(r)}{r}[\sin \theta \cos l \theta(1-\alpha(r))-\cos \theta \sin l \theta] \\
& \frac{\partial \psi_{2}}{\partial x_{1}}=\frac{l f(r)}{r}[\cos \theta \sin l \theta(1-\alpha(r))-\sin \theta \cos l \theta] \\
& \frac{\partial \psi_{2}}{\partial x_{2}}=\frac{l f(r)}{r}[\sin \theta \sin l \theta(1-\alpha(r))+\cos \theta \cos l \theta] \\
& \frac{\partial V_{1}}{\partial x_{1}}=\sin \theta \cos \theta\left[\frac{2 l f(r) \alpha(r)}{r}+\frac{1}{2}\left(f^{2}(r)-1\right)\right] \\
& \frac{\partial V_{1}}{\partial x_{2}}=-l \cos 2 \theta \frac{\alpha(r)}{r^{2}}+\frac{1}{2} \sin ^{2} \theta\left(f^{2}(r)-1\right) \\
& \frac{\partial V_{2}}{\partial x_{1}}=-l \cos 2 \theta \frac{\alpha(r)}{r^{2}}-\frac{1}{2} \cos ^{2} \theta\left(f^{2}(r)-1\right) \\
& \frac{\partial V_{2}}{\partial x_{2}}=-\sin \theta \cos \theta\left[\frac{2 l f(r) \alpha(r)}{r}+\frac{1}{2}\left(f^{2}(r)-1\right)\right]
\end{aligned}
$$

for self-dual ANO vortices with generic (positive) vorticity $l$.

The recurrence formula now gives the local coefficients of the asymptotic expansion in terms of $f(r)$ and $\alpha(r)$, e.g.,

$$
\begin{aligned}
\operatorname{tr}\left[c_{1}\right]\left(\vec{x}, \vec{x} ; \mathcal{H}^{+}\right)= & 5\left[1-f^{2}(r)\right]-\frac{2}{r^{2}} l^{2} \alpha^{2}(r) \\
\operatorname{tr}\left[c_{2}\right]\left(\vec{x}, \vec{x} ; \mathcal{H}^{+}\right)= & \frac{1}{12 r^{4}}\left\{37 r^{4}+4 l^{4} \alpha^{4}(r)+8\left(7 l^{2} r^{2}-8 r^{4}\right) f^{2}(r)+27 r^{4} f^{4}(r)-\right. \\
& \left.-8 l r^{2} \alpha(r)\left[-1+(1+13 l) f^{2}(r)\right]+8 l^{2} \alpha^{2}(r)\left(-2-3 r^{2}+9 r^{2} f^{2}(r)\right)\right\} \\
\operatorname{tr}\left[c_{3}\right]\left(\vec{x}, \vec{x} ; \mathcal{H}^{+}\right)= & \frac{1}{120 r^{6}}\left\{-4 l^{6} \alpha^{6}(r)-4 l^{3} r^{2} \alpha^{3}(r)\left[14+(-132+167 l) f^{2}(r)\right]+4 l^{4} \alpha^{4}(r)\left(20+9 r^{2}+32 r^{2} f^{2}(r)\right)-2 l r^{2} \alpha(r)\left[-4\left(16+9 r^{2}\right)+\right.\right. \\
& \left.+\left(64+96 l-472 l^{2}+344 l^{3}+88 l^{2}+243 l r^{2}\right) f^{2}(r)+(-52+109 l) r^{2} f^{4}(r)\right]+l^{2} \alpha^{2}(r)\left[-256-144 r^{2}-117 r^{4}+\right. \\
& \left.+2 r^{2}\left(88-548 l+516 l^{2}+183 r^{2}\right) f^{2}(r)+99 r^{4} f^{4}(r)\right]+r^{2}\left[r^{2}\left(-16+151 r^{2}\right)+\left(-320 l^{3}+160 l^{4}+32 r^{2}+48 l r^{2}-\right.\right. \\
& \left.\left.\left.-321 r^{4}+8 l^{2}\left(20+39 r^{2}\right)\right) f^{2}(r)+r^{2}\left(-16-48 l+44 l^{2}+199 r^{2}\right) f^{4}(r)-29 r^{4} f^{6}(r)\right]\right\}
\end{aligned}
$$


We have explicitly given only the first three local coefficients of the heat kernel expansion for $\mathcal{H}^{+}$because the complexity of the expressions increases with $n$ enormously. Additionally,

$$
\begin{aligned}
& c_{1}\left(\vec{x}, \vec{x} ; \mathcal{H}^{G}\right)=1-f^{2}(r) \\
c_{2}\left(\vec{x}, \vec{x} ; \mathcal{H}^{G}\right)= & \frac{-1}{6 r^{2}}\left\{\left[4 l^{2}+5 r^{2}-8 l^{2} \alpha(r)+4 l^{2} \alpha^{2}(r)\right] f^{2}(r)+\right. \\
& \left.-3 r^{2}-2 r^{2} f^{4}(r)\right\} \\
c_{3}\left(\vec{x}, \vec{x} ; \mathcal{H}^{G}\right)= & \frac{1}{60 r^{4}}\left\{10 r^{4}-\left[-32 l^{3}+16 l^{4}+8 l r^{2}+23 r^{4}+\right.\right. \\
+16 l^{2}\left(1+r^{2}\right)- & 8 l\left(-12 l^{2}+8 l^{3}+r^{2}+4 l\left(1+r^{2}\right)\right) \alpha(r)+ \\
+16 l^{2}\left(1-6 l+6 l^{2}+\right. & \left.r^{2}\right) \alpha^{2}(r)+32(1-2 l) l^{3} \alpha^{3}(r)+ \\
\left.+16 l^{4} \alpha^{4}(r)\right] f^{2}(r)+ & r^{2}\left[8 l+16 l^{2}+17 r^{2}+16 l^{2} \alpha^{2}(r)-\right. \\
& \left.-8 l(1+4 l) \alpha(r)] f^{4}(r)-4 r^{4} f^{6}(r)\right\}
\end{aligned}
$$

are the first three local coefficients for the heat kernel expansion for the ghost operator $\mathcal{H}^{G}$.

Plugging these expressions into the partially analytical partially numerical solution for $f(r)$ and $\alpha(r)$, it is possible to compute the local coefficients and integrate them numerically over the whole plane.

\section{Mass shift for vorticities $l=1, l=2, l=3, l=4$}

Finally, the one-loop quantum correction of the vortex solution with vorticity $l$ is given by formula (19)

$$
\Delta M_{V}=-\frac{\hbar m}{2}\left[\frac{1}{8 \pi \sqrt{\pi}} \sum_{n=2}^{N_{0}} \bar{c}_{n} \gamma\left[n-\frac{3}{2}, 1\right]+\frac{2 l}{\sqrt{\pi}}\right]
$$

Using the Mathematica environment in a modest $\mathrm{PC}$ we have obtained the coefficients shown in Tables IV and V,

TABLE IV: Seeley Coefficients for $l=1,2$.

\begin{tabular}{|c|cc|cc|}
\hline & \multicolumn{2}{|c|}{$l=1$} & \multicolumn{2}{c|}{$l=2$} \\
\hline$n$ & $c_{n}\left(\mathcal{H}^{+}\right)$ & $c_{n}\left(\mathcal{H}^{G}\right)$ & $c_{n}\left(\mathcal{H}^{+}\right)$ & $c_{n}\left(\mathcal{H}^{G}\right)$ \\
\hline 2 & 30.36316 & 2.60773 & 61.06679 & 6.81760 \\
3 & 12.94926 & 0.31851 & 25.61572 & 1.34209 \\
4 & 4.22814 & 0.022887 & 8.21053 & 0.20481 \\
5 & 1.05116 & 0.0011928 & 2.02107 & 0.023714 \\
6 & 0.20094 & 0.00008803 & 0.40233 & 0.002212 \\
\hline
\end{tabular}

We remark that formula (19) depends on the number $N_{0}$ chosen to cut the asymptotic heat kernel expansions. We have no means of determining the optimum value for $N_{0}$, but in practice we can only cope with a small $N_{0}$ value; a big $N_{0}$ would require the computation of
TABLE V: Seeley Coefficients for $l=3,4$.

\begin{tabular}{|c|cc|cc|}
\hline & \multicolumn{2}{|c|}{$l=3$} & \multicolumn{2}{c|}{$l=4$} \\
\hline$n$ & $c_{n}\left(\mathcal{H}^{+}\right)$ & $c_{n}\left(\mathcal{H}^{G}\right)$ & $c_{n}\left(\mathcal{H}^{+}\right)$ & $c_{n}\left(\mathcal{H}^{G}\right)$ \\
\hline 2 & 90.20440 & 11.51035 & 118.67540 & 16.46895 \\
3 & 36.68235 & 2.60898 & 46.01141 & 4.00762 \\
4 & 11.69979 & 0.46721 & 14.64761 & 0.77193 \\
5 & 2.86756 & 0.067279 & 3.58906 & 0.11747 \\
6 & 0.566227 & 0.0079269 & 0.667202 & 0.01620 \\
\hline
\end{tabular}

an enormous number of local coefficients. Nevertheless, the choice $N_{0}=6$ is acceptable. The behavior of the asymptotic series in (19) is given in Table VI:

TABLE VI: Convergence of the asymptotic series in units of $\hbar m$.

\begin{tabular}{|c|cccc|}
\hline$N_{0}$ & $\Delta M_{V}\left(N_{0}\right)$ & $\Delta M_{V}\left(N_{0}\right)$ & $\Delta M_{V}\left(N_{0}\right)$ & $\Delta M_{V}\left(N_{0}\right)$ \\
& $l=1$ & $l=2$ & $l=3$ & $l=4$ \\
\hline 2 & -1.02951 & -2.03787 & -3.01187 & -3.97025 \\
3 & -1.08323 & -2.14111 & -3.15680 & -4.14891 \\
4 & -1.09270 & -2.15913 & -3.18208 & -4.18014 \\
5 & -1.09427 & -2.16212 & -3.18628 & -4.18534 \\
6 & -1.09449 & -2.16257 & -3.18690 & -4.18606 \\
\hline
\end{tabular}

The convergence up to the sixth order in the asymptotic expansion is very good. In the case of $\lambda(\phi)_{2}^{4}$ kinks we found agreement between the result obtained by this method and the exact result up to the fourth decimal figure, see [9], by choosing $N_{0}=10$.

There are reasons to expect this behavior on general analytical grounds. Truncation of the asymptotic expansion of the heat function at order $N_{0}$ produces an error of order $\beta^{N_{0}}$, which in turn leads to an error proportional to $\gamma\left[N_{0}-\frac{1}{2}, 1\right] \simeq \frac{1}{N_{0}-\frac{1}{2}}$, for $N_{0}$ large, in the computation of the $\zeta_{\mathcal{H}^{+}}\left(-\frac{1}{2}\right)$ zeta function, see 22] Section 1.10. In fact, the rate of convergence is improved in our problem by the the smallness of the $c_{n}$ coefficients, see Tables IV and $\mathrm{V}$, for large $\mathrm{n}$. This smallness is due to the fact that, when $n$ increases, higher and higher powers of partial derivatives of the field profiles of increasing order enter in the computation of $c_{n}$. The vortex solutions, however, are as regular and smooth as allowed by the topology. Therefore, the admitted error by cutting the mass shift formula at $N_{0}=6$ is especially small for low vorticities.

In Table VII we give the one-loop quantum corrections for the vortex solutions up to $l=4$, whereas we plot the correction in the figure as a function of the magnetic flux. The broken line (linear function) represents the hypothetical situation in which each magnetic flux quantum would contribute with the same correction. Hence, this is almost - within the error margin- the situation that we 
TABLE VII: One-Loop Quantum Mass Correction to the vortex with vorticity $l=1,2,3,4$.

\begin{tabular}{|l|l|}
\hline$l$ & $\Delta M_{V} / \hbar m$ \\
\hline 1 & -1.09449 \\
2 & -2.16257 \\
3 & -3.18690 \\
4 & -4.18606 \\
\hline
\end{tabular}

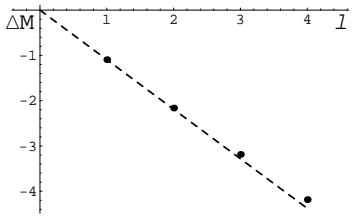

have found.

These results, however, do not allow us to answer the question of whether or not the classical degeneracy with respect to the vortex centers observed at the classical level also holds at one-loop order. The figure in Table VII seems to suggest that the mass shift of $l$ well separated vortices is equal -modulo errors- to $l$ times the mass shift of a single vortex, but we do not know in what direction the errors run.

\section{Mass shift for solutions with two separate vortices}

We now offer two Tables, VIII and IX, where Seeley coefficients and the quantum corrections are given for two-vortex solutions with intermediate separations $d=1$, $d=2$, and $d=3$ between superimposed vortices, $\omega=0$ in (10)-(11), and well separated vortices, $\omega=1$ in (10)(11). The coefficients of the asymptotic expansion are computed only up to third order because much more computation time is required. Also, we stress that in this situation, with no cylindrical symmetry, we expect not so good results because there are two more important sources of errors: first, the variational solutions with two separate vortices are far less exact than the solution with $l=2$ and cylindrical symmetry. Second, even though another numerical method would be used in the search of vortex solution we would run in difficulties; there is no way to avoid the use of partial derivatives in the calculation of the coefficients because the vortex equations alone are not enough.

TABLE VIII: Seeley Coefficients for $d=1,2,3$.

\begin{tabular}{|c|c|c|c|c|c|}
\hline & \multicolumn{2}{|c|}{$d=1$} & \multicolumn{2}{|c|}{$d=2$} & $d=3$ \\
\hline$n$ & $c_{n}\left(\mathcal{H}^{+}\right)$ & $c_{n}\left(\mathcal{H}^{G}\right)$ & $c_{n}\left(\mathcal{H}^{+}\right)$ & $c_{n}\left(\mathcal{H}^{G}\right)$ & $c_{n}\left(\mathcal{H}^{+}\right) c_{n}\left(\mathcal{H}^{G}\right)$ \\
\hline 2 & 61.0518 & 6.81277 & 58.3359 & 6.46609 & $57.3420 \quad 6.03872$ \\
\hline 3 & 25.6137 & 1.33822 & 24.5050 & 1.23466 & 24.11871 .02031 \\
\hline
\end{tabular}

TABLE IX: Convergence of the asymptotic series.

\begin{tabular}{|c|ccc|}
\hline$N_{0}$ & $\Delta M_{V}\left(N_{0}\right) / \hbar m$ & $\Delta M_{V}\left(N_{0}\right) / \hbar m$ & $\Delta M_{V}\left(N_{0}\right) / \hbar m$ \\
& $d=1$ & $d=2$ & $d=3$ \\
\hline 2 & -2.03770 & -1.99798 & -1.98848 \\
3 & -2.14095 & -2.09695 & -2.08672 \\
\hline
\end{tabular}

\section{SUMMARY AND OUTLOOK}

The one-loop mass shifts of superimposed vortices with low magnetic fluxes are:

$$
\begin{aligned}
& M_{V}^{l=1}=m\left(\frac{\pi v}{e}-1.09427 \hbar\right)+o\left(\hbar^{2}\right) \\
& M_{V}^{l=2}=2 m\left(\frac{\pi v}{e}-1.08106 \hbar\right)+o\left(\hbar^{2}\right) \\
& M_{V}^{l=3}=3 m\left(\frac{\pi v}{e}-1.06230 \hbar\right)+o\left(\hbar^{2}\right) \\
& M_{V}^{l=4}=4 m\left(\frac{\pi v}{e}-1.04651 \hbar\right)+o\left(\hbar^{2}\right) .
\end{aligned}
$$

Much less precise results are also provided for twovortices with separate centers. This is to be compared with the supersymmetric result:

$$
\mathcal{M}_{V}^{l}=|l| m\left(\frac{\pi v}{e}-0.5000 \hbar\right)+o\left(\hbar^{2}\right)
$$

see [4] and [5]. We notice that the one-loop correction due to bosonic fluctuations of self-dual vortices is almost twice the correction arising in the supersymmetric system coming only from mass renormalization counterterms when proper SUSY-preserving boundary conditions are imposed. The same proportion holds between one-loop corrections to sine-Gordon and $\phi^{4}$ kink masses in the non-supersymmetric and supersymmetric frameworks, see [14] and 15].

It seems plausible that a similar method can successfully be applied to compute the one-loop mass shift for self-dual Chern-Simons-Higgs vortices, see 20]-21]. A Hamiltonian formalism in the topological sectors of the first-order CSH Lagrangian system should be first developed. More ambitious, generalized zeta functions of $12 \times 12$ matrix PDE operators in three variables are essential in computing the one-loop mass shift to BPS monopoles. Thus, our procedure opens a door to calculate quantum corrections to BPS monopole masses in a $\mathcal{N}=0$ bosonic setting to be contrasted with the $\mathcal{N}=2$ and $\mathcal{N}=4$ supersymmetric results of [8] and [26].

\section{Acknowledgements}

Partially financed by the Spanish Ministerio de Educacion y Ciencia under grant: BFM2003-00936 
[1] A. Alonso Izquierdo, W. Garcia Fuertes, J. Mateos Guilarte and M. de la Torre Mayado, Phys. Rev. D70 (2004) 061702(R).

[2] J.R. Schmidt, Phys. Rev. D46 (1992) 1839.

[3] B.H. Lee and H. Min, Phys. Rev. D51 (1995) 4458.

[4] D.V. Vassilevich, Phys. Rev. D68 (2003) 045005.

[5] A. Rebhan, P. van Nieuwenhuizen, and R. Wimmer, Nucl. Phys. B679 (2004) 382.

[6] H. Nastase, M. Stephanov, A. Rebhan and P. van Nieuwenhuizen, Nucl. Phys. B542 (1999) 471.

[7] M. Shifman, A. Vainshtein and M. Voloshin, Phys. Rev. D59(1999) 045016.

[8] A. Rebhan, P. van Nieuwenhuizen, and R. Wimmer, Phys. Lett. B594 (2004) 234.

[9] A. Alonso Izquierdo, W. Garcia Fuertes, M. A. Gonzalez Leon and J. Mateos Guilarte, Nucl. Phys. B635 (2002) 525.

[10] M. Bordag, A. Goldhaber, P. van Nieuwenhuizen and D. Vassilevich, Phys. Rev. D66 (2002) 125014.

[11] R. Dashen, B. Hasslacher and A. Neveu, Phys. Rev. D10 (1974) 4130.

[12] A. Alonso Izquierdo, W. Garcia Fuertes, M. A. Gonzalez Leon and J. Mateos Guilarte, Nucl. Phys. B638 (2002) 378.

[13] A. Alonso Izquierdo, W. Garcia Fuertes, M. A. Gonzalez Leon and J. Mateos Guilarte, Nucl. Phys. B681 (2004) 163.

[14] J.F. Schonfeld, Nucl. Phys B161 (1979) 125.
[15] R. Wimmer, Quantization of supersymmetric solitons, hep-th/0109119

[16] M. Veltman, Reflections on the Higgs system, CERN Yellow Report 97-05, 1997

[17] H. J. de Vega and F. A. Schaposnik, Phys. Rev. D14 (1976) 1100.

[18] L. Jacobs and C. Rebbi, Phys. Rev. B19 (1979) 4486

[19] E. Weinberg, Phys. Rev. D19 (1979) 3008.

[20] R. Jackiw, K. Lee and E.J. Weinberg, Phys. Rev. D42 (1990) 3488

[21] W. Garcia Fuertes and J. Mateos Guilarte, Eur. Phys. J. C9 (1999) 167.

[22] P. Gilkey, Invariance theory, the heat equation and the Atiyah-Singer index theorem, Publish or Perish, Delaware, (1984).

[23] J. Roe, Elliptic operators, topology and asymptotic methods Longman Scientific and Technical, New York (1988).

[24] A. Jaffe and C.H. Taubes, Vortices and monopoles, Birkhauser, (1980).

[25] A. Rebhan and P. van Nieuwenhuizen, Nucl. Phys. B508 (1997) 449.

[26] A. Rebhan, R. Schoefbeck, P. van Nieuwenhuizen, R. Wimmer, BPS saturation of the $\mathcal{N}=4$ monopole by infinite composite-operator renormalization, hep-th/0502221

[27] In [4] there is the following footnote: "Since no analytic form for the profile functions of the ANO vortices is available, calculations of the mass shift in a nonsupersymmetric case is a rather complicated problem". 